\title{
Combinatorial synthesis approach employing graph networks
}

\author{
Offer Shai ${ }^{a, *}$, Noel Titus ${ }^{b}$, Karthik Ramani ${ }^{b}$ \\ ${ }^{a}$ Mechanical Engineering School, Tel-Aviv University, Israel \\ ${ }^{\mathrm{b}}$ School of Mechanical Engineering, Purdue University, West Lafayette, IN, USA
}

Received 17 August 2007

\begin{abstract}
The paper proposes a methodology to assist the designer at the initial stages of the design synthesis process by enabling him/her to employ knowledge and algorithms existing in graph network theory. The proposed method comprises three main stages: transforming the synthesis problem into a graph theoretic problem; devising the topology possessing special engineering properties corresponding to the system requirements; finding the geometric configuration of that topology that will possess the desired properties. To clarify the idea and to demonstrate its generality, the approach is presented through three synthesis case studies from different engineering domains: electrical networks, statics and kinematics.

As is highlighted in the paper, the approach of employing graph theory in the synthesis process offers several unique advantages. Among these advantages are: gaining a general perspective on different synthesis problems from different engineering domains by transforming them into the same graph problem; employing the same graph algorithms for different synthesis problems; establishing the existence of configurations with special properties solely from the topology of the system; transferring knowledge and methods between different engineering disciplines for both the topology and the geometry generation steps.
\end{abstract}

(c) 2007 Elsevier Ltd. All rights reserved.

Keywords: Combinatorial synthesis; Graph theory; Assur groups; Self-dual graphs; Statics; Kinematics; Electrical circuits

\section{Introduction}

Design synthesis of engineering artifacts has been a significant research area, especially in the fields of structures $[4,24,25,9]$, mechanical systems [26,16,8,6,7], MEMS and VLSI - a good summary of which is available in [1]. Techniques ranging from search algorithms, stochastic and gradient based optimization, evolutionary and genetic algorithms, have been used in combination with discrete and continuum approaches, bond graph methods, graph grammars, design rules and more for the purpose of synthesis of these systems.

However, as is evident from the publications cited above and others, no universal or common synthesis method has been applied to different engineering domains. Each has its own distinct approach that utilizes the properties of the

\footnotetext{
* Corresponding author. Tel.: +972 3640 8158; fax: +97236407617.

E-mail address: shai@eng.tau.ac.il (O. Shai).
}

engineering domain, through a synthesis technique suited to that domain.

The current paper proposes a common representation method applicable for different engineering systems, rendering a possibility for devising a standard synthesis process for a multi-domain engineering system. The method employs graph theoretical models to transform the design problem from the engineering domain to the domain of discrete mathematics. The engineering design problems of various engineering domains then become problems in graph theory, solutions of which may employ the variety of combinatorial algorithms developed in the field. A similar approach has already been employed for developing advanced engineering analysis techniques, as reported in $[14,17,11,21]$ and more, while the current paper focuses entirely on the issues of synthesis.

The outline of the paper is as follows.

Section 2 describes the steps in the proposed approach followed in the paper. It is explained how a synthesis 
problem is transformed into terms of graphs and then solved through known graph-theoretic theorems and algorithms. This process is then followed by the topology generation of the physical system that possesses unique properties relevant to the original synthesis problem.

Section 3 introduces the notion of using a common representation schema for different engineering domains through examples in structures, kinematics and electrical circuits.

This is followed up in Section 4 with an explanation of how systems in different engineering domains can be represented in the common mathematical language of graph theory and shows a further elucidation of the examples introduced in Section 3.

Section 5 describes the process of synthesizing a solution to a given engineering problem starting with a root graph structure. This section highlights one of the unique properties of this approach in which the same algorithm can be applied for solving problems from different engineering domains.

Section 6 employs combinatorial methods to characterize the configurations of engineering systems possessing the special engineering properties that correspond to the system requirements. An example in structures is used to demonstrate how the combinatorial approach goes beyond topology, to incorporate also geometrical treatment of the problems.

\section{General description of the proposed combinatorial synthesis method}

The method introduced in this paper consists of three main stages (Fig. 1): transforming the synthesis problem into a problem in graph theory; devising the proper topology which possesses the required engineering property; the configuration synthesis step, which involves finding the geometry that exhibits the desired engineering property. A notable feature of this approach is that during the synthesis process, the engineering problems are transformed

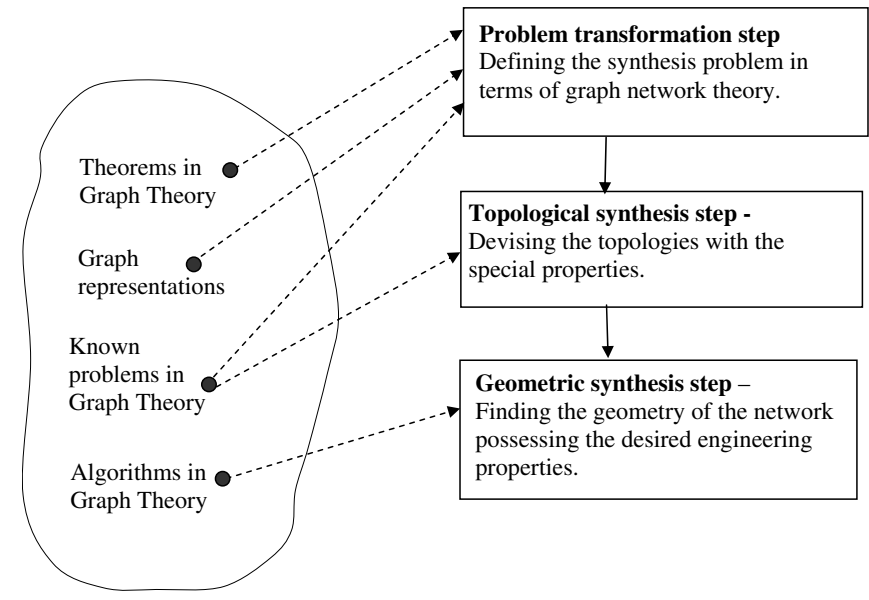

Fig. 1. Main aspects of the proposed synthesis method and the mathematical steps employed throughout the proposed synthesis process. into known problems in graph theory, the solutions of which can be obtained from existing graph theory algorithms.

Fig. 1 presents the flow diagram for the suggested synthesis approach in a top down manner, while outlining the mathematical foundation each level requires. The right side of the figure lists the three main stages, while the left side of the figure lists the mathematical subjects employed through the process. The dashed arrows connecting the left and the right sides of the figure indicate which mathematical subjects are employed at each step of the proposed synthesis procedure.

The mathematical subjects from graph theory that are employed in the synthesis process are as follows:

1. Theorems in graph theory - a system of theorems, axioms and rules developed in graph theory underlying the mathematical behavior of graphs [10].

2. Graph representations - graphs augmented by additional mathematical variables and properties. The variables and properties associated with such representations are employed in mapping the variables and properties underlying the physical behavior of engineering systems. As is explained in [21], different sets of the augmented variables and their properties yield different types of graph representations. The basic idea of replacing the treatment of an engineering system with the treatment of a network graph is thoroughly elaborated in $[3,14,11,17]$.

3. Known problems in graph theory - as any other mathematical field, graph theory is characterized by the set of problems treated through its mathematical apparatus [10]. From the point of view of the current paper, the problem in graph theory, once converted from the engineering domain, is seen as a problem fully formulated in the terminology of combinatorial mathematics. The known graph theory problems, or at least their special cases, are treatable through known graph-theoretical tools, as described below.

4. Algorithms in graph theory - known methods and algorithms developed to solve problems in graph theory $[10,15]$.

The stages of the proposed synthesis methodology that are supported by the above mathematical foundation are described as follows:

1. Problem transformation step - This step involves the formulation of the transformed engineering synthesis problem to a purely mathematical problem in graph theory. The transformation is performed through the transformation rules used when constructing the graph representation for the engineering system [21,3]. The type of graph representation chosen depends both on the engineering domain for which the original synthesis problem has been formulated and on the nature of the specific design requirements and constraints. 
Once the problem formulation in the terminology of graph theoretic form has been obtained, the problem is correlated with known graph theoretical problems. If such a correlation is not straightforward, one may employ known theorems of graph theory as intermediate steps in the problem formulation until a known problem in graph theory is obtained.

2. Topological synthesis step - At this stage, graph theoretical algorithms are employed to devise the solution to the problem formulated in the previous stage. As will be illustrated by the synthesis problems in the current paper, the graph theoretic equivalent of an engineering problem will in most cases be a graph topology satisfying a set of mathematical requirements. Some of the problems will involve developing a topology from scratch, while others require no more than adjusting a given topology.

3. Geometric synthesis step - The final step bridges the gap between the found topology of a system and its design description. In some cases when the engineering domain is one dimensional or scalar, such as in electric networks, this step is irrelevant and can be omitted. In cases of multidimensional systems such as structures, their geometrical properties are mapped as the vector variables and constant properties associated with the elements of the corresponding graph representation. As is shown later in the paper, determining or adjusting these vector properties and variables can be achieved by employing algorithms known in graph theory.

As can be seen, the initial steps of the synthesis methodology shown here are based on graph theory entities, while the later steps involve the engineering elements. The last step deals exclusively with geometry and configuration issues.

The proposed approach can be used both as stand alone (as is demonstrated in the case studies shown in the paper), but for higher effectiveness can also be used in combination with known engineering design techniques. For instance, at the stage of topology generation one can be aided by stochastic methods such as genetic algorithms and simulated annealing $[13,6,7]$. Alternatively, introducing new connections and joints in a systematic manner during topology generation is a challenge, primarily due to the dynamic nature of the problem, as pointed out by [19]. The graph theoretic approach allows for a dynamic representation of the topological synthesis problem. Additionally, since specific engineering properties are utilized in generating new topologies, the topology synthesis problem is a systematic procedure. Hence, in the approach presented in the paper, both the topological synthesis and geometrical synthesis steps are amenable to linking with evolutionary optimization methods.

\section{The synthesis case studies used in the paper}

In this section three synthesis problems are introduced while their solutions are shown in the subsequent sections, using the approach described in Section 2. To highlight the generality of the approach, the problems were chosen from three different engineering domains: electrical networks, structural mechanics and kinematics. In addition, the solutions of these three problems will demonstrate that new relations between different engineering domains can be revealed, so that the same algorithms can be applied to solve problems in different engineering domains. It should be pointed out that, of the presented examples, only the two problems from statics and kinematics involve geometrical considerations, while the electrical problem is onedimensional.

\subsection{Synthesis of multi-functional electrical networks}

The first case study is the synthesis of an equivalent electrical circuit that exhibits the property of interchangeability between the voltages and currents of the original circuit with the currents and voltages, respectively, of the equivalent circuit. In these specific electrical circuits, when the voltage and current sources are switched and the resistances become conductivities with the same values, a special property is revealed, namely the voltage behavior of the elements in the original circuit becomes identical to the current behavior of the elements in the second circuit.

Fig. 2 depicts two circuits where the first one (Fig. 2a) possesses the above property while the seemingly similar circuit in Fig. $2 \mathrm{~b}$ does not. The synthesis procedure to construct such types of electrical circuits is given in Section 5.1. a

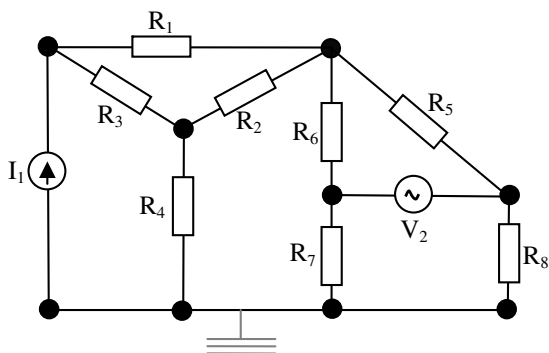

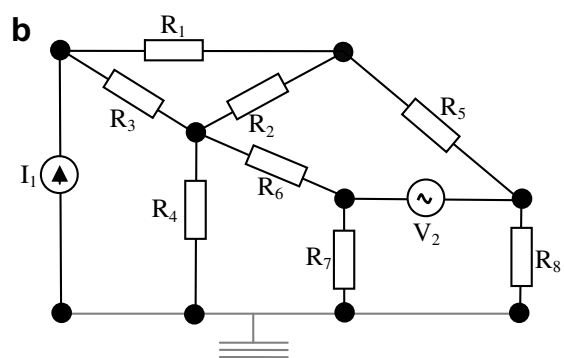

Fig. 2. Example of an electrical circuit that possesses the interchangeability property between voltages and currents (a) and one that does not (b). 


\subsection{Synthesis of trusses with special geometrical properties}

In the previous problem we referred to the synthesis of networks that possess a special topological property. In the second problem, we extend this methodology and introduce a way to construct a topology that possesses special geometrical properties. In other words, this approach is directed towards reasoning as to whether a given topology after realization may have a geometric configuration with a special engineering property both in statics and kinematics.

Specifically, in the domain of structures, for example, by using the proposed methodology it will be shown how to conclude from the topology of a structure whether there exists a geometric configuration in which an internal force in one of its bars exerts forces in all the other bars and in which all the inner joints have infinitesimal motions. In the paper, this property will be referred to as the "self-stress mobility property". Using this property it will be possible to conclude that the truss in Fig. 3a possesses this property, while the truss in Fig. 3b, since only joint B of the truss can move, does not possess the self-stress mobility property.

In the following sections it will be shown how topologies of trusses possessing the self-stress mobility property can be constructed.

Fig. 4a depicts a topology of a truss, for which there exist numerous configurations possessing the self-stress mobility property. On the other hand, for the topology of Fig. 4b there is no configuration with this property for any choice of the lengths of the bars or their inclinations.

\subsection{Synthesis of kinematic linkages with a special locking position property}

The third synthesis example exploits another unique feature of the combinatorial synthesis approach which enables utilization of solutions to engineering problems in one engineering domain to synthesize solutions in another engineering domain. This is done by performing transformations on the graph network representing the system in the original engineering domain. This idea is illustrated by transforming the results obtained in the previous synthesis problem in statics, into a different engineering domain, namely kinematics. a

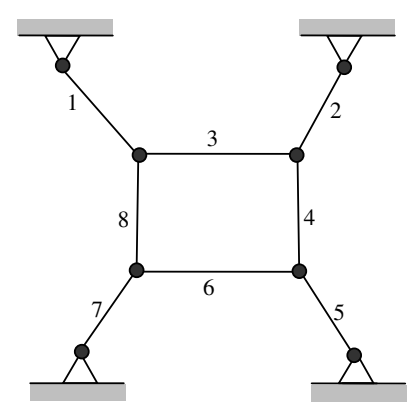

b

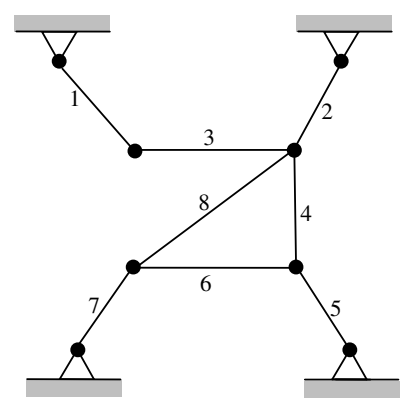

Fig. 4. The topology of a static truss for which there exist configurations with the self-stress mobility property (a) and the topology of the truss for which there is no such configuration (b).

In the current kinematic synthesis problem it is desired to construct topologies of linkages for which there exists at least one configuration in a locking position which possesses two properties:

- First property: The configuration will be such that the force exerted by the driving link is resisted by forces in all the other links of the system.

- Second property: Any of the links in the linkage should be able to drive the linkage out of the locked position.

These two properties are important in a variety of applications, including the synthesis of deployable structures [12].

Fig. 5 depicts two kinematic systems; the left one (a) does not possess the above properties while the right one (b) does. The topological reasoning and the algorithm to construct such kinematic systems appear in Section 5.3.

In the next section the method of transforming the synthesis problem into a problem in network graph theory will be explained.

\section{Transforming the synthesis problems into graph theory problems}

This initial step transforms the synthesis problem from an engineering domain into a graph theory problem,

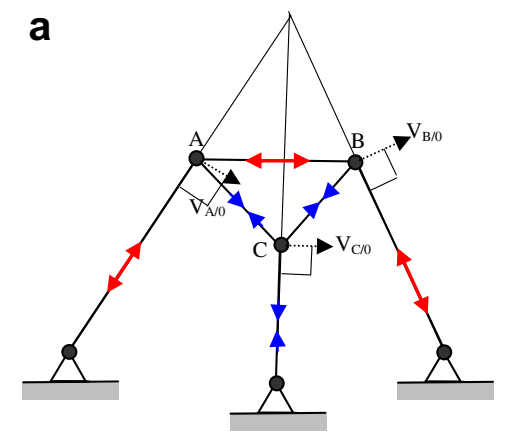

b

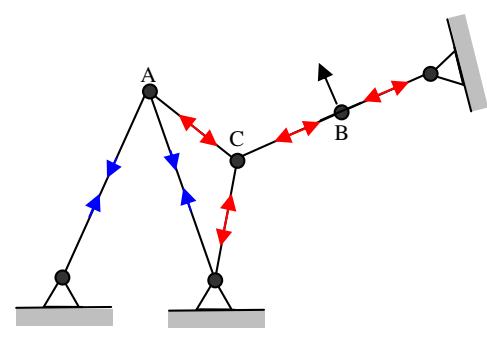

Fig. 3. The configuration of a truss (a) that has the self-stress mobility property while the configuration in (b) does not. 


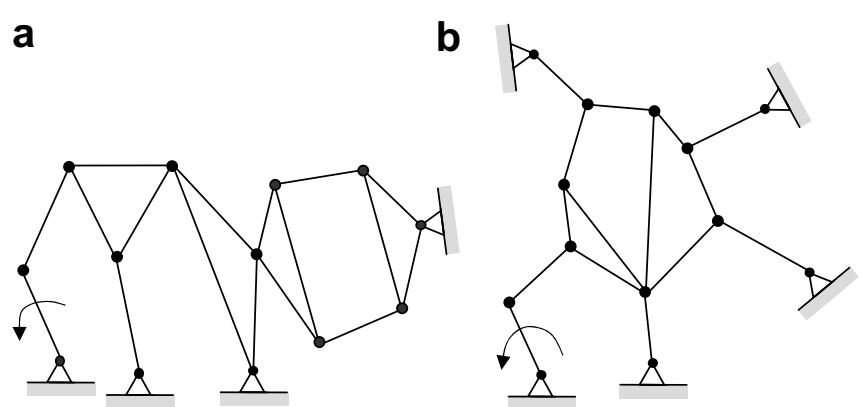

Fig. 5. Kinematic linkage that does not have the special locking property (a) and one that does (b).

enabling the use of knowledge and algorithms that exist in graph and network theories to solve the engineering synthesis problems. In many cases, different synthesis problems become the same graph problem, enabling the same algorithm to be employed to solve different problems that are currently considered to be completely unrelated.

Although the synthesis problems in this paper belong to three different engineering domains, the algorithms for solving them will be shown to be similar. This opens up a new way for transforming knowledge between engineering domains and obtaining a general perspective of the synthesis problem.

\subsection{The multi-functional electrical synthesis problem in graph terminology}

Throughout the paper, particularly in this section, we use one of the basic topics in graph theory - graph duality. For more details on graph duality the readers are referred to introductory textbooks on graph theory, such as [10].

For every planar graph $G=(V, E)$ (i.e., a graph that can be drawn in the plane in such a manner that its edges do not intersect), there exists another graph, $G^{*}$, termed a dual graph that is constructed as follows: for every edge in the original graph there is a corresponding edge in the dual graph and for every face (circuit without inner edges) in the original graph there exists a vertex in the dual graph. Any two vertices are adjacent in the dual graph if and only if the corresponding faces in the original graph are adjacent. For example, in Fig. 6, the faces defined by links
$\{1,2\}$ and $\{2,3,5\}$ are adjacent in the original graph (Fig. 6a), thus the corresponding vertices $\mathrm{A}$ and $\mathrm{B}$, respectively, are adjacent in the dual graph (Fig. 6b) on the same edge.

The duality between the graphs applies also to the elements of the graph. For example, the edges constituting a cutset in the original graph (a set of edges whose removal disconnects the graph) correspond to edges that constitute a circuit in the dual graph. For example, the edges $\{1,2,5\}$ in the original graph, Fig. 6a, form a cutset while the corresponding edges in the dual graph $-\left\{1^{\prime}, 2^{\prime}, 5^{\prime}\right\}$ form a circuit (Fig. 6b).

When dealing with the engineering domain of electrical circuits, the cutset equations govern the behavior of the electric currents (Kirchhoff's Current Law) and the circuit equations govern the behavior of the voltages (Kirchhoff's Voltage Law). By means of the duality relation, the current equations in the original graph are identical to the voltage equations in the dual graph [3].

Since we are looking for circuits in this synthesis case for which there exists a correspondence between the currents and voltages, we need to construct them so that their corresponding graphs are dual to themselves. Following this topological rule it can be verified that the known diode bridge rectifier circuit (Fig. 7a) is multi-functional, i.e., it can serve as a rectifier of both voltages and currents while another circuit, the full wave rectifier (Fig. 7b), does not possess this property as it rectifies only voltages.

\subsection{The synthesis of trusses with self-stress mobility property in graph theory terms}

The case study presented in this and the following sections possess a clear distinction from the multi-functional electrical networks described in the previous section. As the electrical circuits lack geometrical information, determining the system topology is sufficient for devising the design description. On the other hand, when dealing with multidimensional systems, such as structures and kinematical systems, the geometrical information is essential and an additional step - the geometric synthesis step is being used.

In the previous case study the topological synthesis was transformed into a search for a specific class of graphs
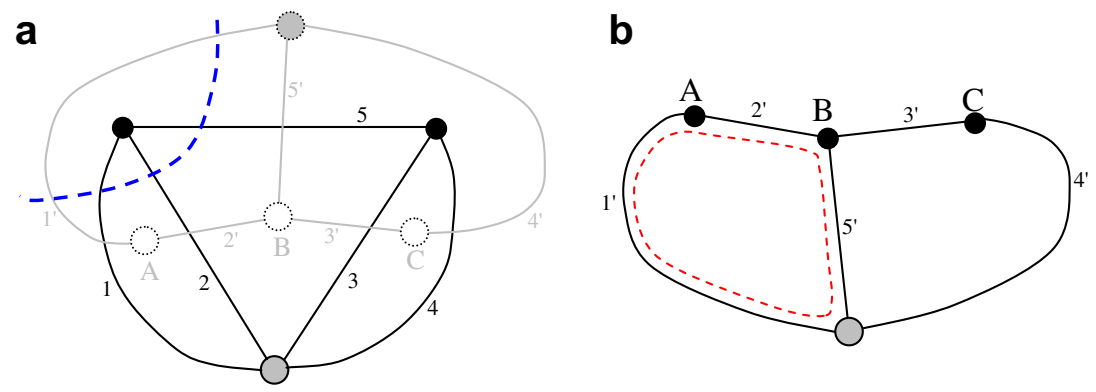

Fig. 6. The correlation between the original graph and its dual graph. (a) The original graph and the highlighted cutset (in blue). (b) The dual graph and the highlighted dual circuit (in red). 

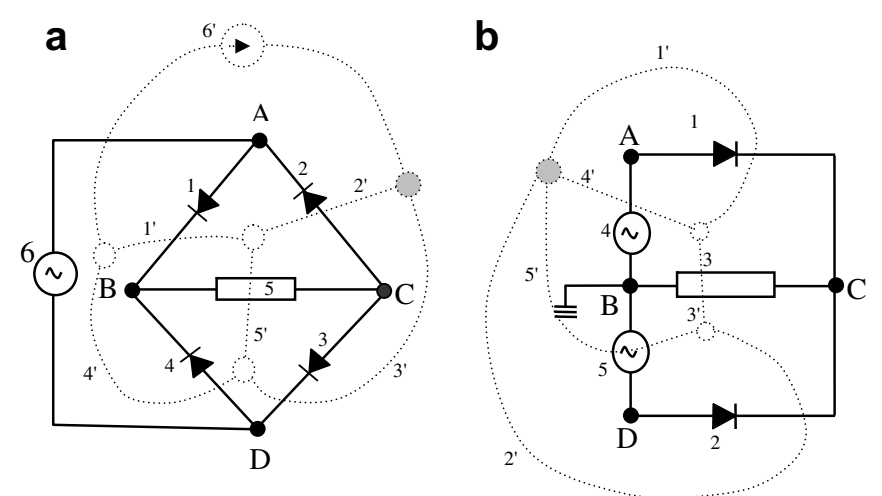

Fig. 7. Example of a diode bridge rectifier circuit that is self-dual (a) and the full wave rectifier circuit that is not (b).

called self-dual graphs. In the current case study the search for the proper topology is performed in a sub-class of a known class of graphs. In general, computational resources needed to find the proper topology can be reduced when graphs belonging to specific classes are utilized in synthesis, since properties associated with those classes of graphs can be taken advantage of.

Since this topological synthesis deals with trusses with a specific property, we need to search for a class of graphs corresponding to trusses which are rigid, i.e., any generic realization of the graphs the trusses will be stable. This class of graphs is called "Laman graphs" [15] and is defined by the following criteria:

1. $e(G)=2^{*} v(G)-3$, where $e(G)$ and $v(G)$ stands for the number of edges and vertices in graph $G$, respectively.

2. All the subgraphs, $G^{\prime} \subseteq G$ satisfy the relation: $e\left(G^{\prime}\right) \leqslant 2^{*} v\left(G^{\prime}\right)-3$.

For example, the graph in Fig. $8 \mathrm{~b}$ is not a Laman graph since the subgraph defined by the set of vertices $\{B, C, D, E\}$ does not satisfy condition 2 .

The search for finding a proper truss possessing the stability and self-stress mobility properties, becomes a search for a graph among a desired set of graphs. This set is defined to be the intersection of Laman graphs, those graphs whose realization is always stable, corresponding to the stability property, with the set of self-dual graphs, corresponding to the property of the self-stress mobility. This desired set of graphs is depicted in Fig. 9 as the inter-
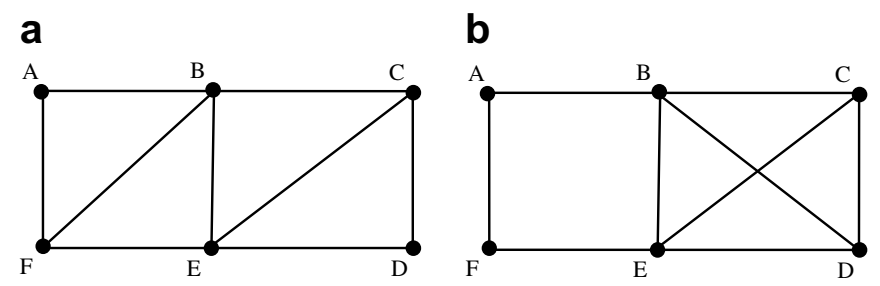

Fig. 8. Example of a graph satisfying both Laman graph criteria (a) and the one that satisfies only criterion 1 (b).

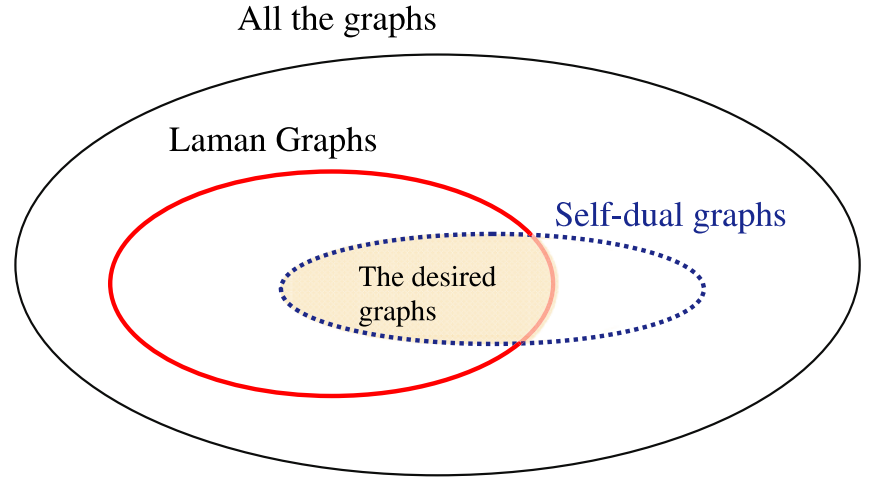

Fig. 9. The hierarchic order showing the relation between the desired graphs, the graphs corresponding to the trusses with the self-stress mobility property, and other possible graph families.

section of the two graph sets and is designated with a gray color.

Recently, it has been noticed that special schematic structures developed for kinematic systems, termed Assur Groups [2] are actually a special class of Laman graphs [22]. These graphs are defined in graph terminology as minimal rigid in relation to vertices, i.e., deleting any set of vertices results in a graph that is not rigid.

It has been mathematically established [20] on the basis of a number of conjectures [22] that all the Assur graphs and only Assur graphs possess configurations with the self-stress mobility property. In other words, if a given configuration has this property it is mathematically proven that its topology is an Assur graph.

For example, the truss in Fig. 10a is an Assur graph while that in Fig. 10b is not, since after deleting the vertices $\{\mathrm{D}, \mathrm{E}, \mathrm{F}, \mathrm{G}\}$ the remaining truss is rigid. Thus, from the above theorem it follows that only for the truss appearing in Fig. 10a it is possible to find a configuration having that property.

\subsection{The synthesis of linkages with special locking position property in graph theory terms}

Section 4.2 outlined the discovery of a new sub-class of graphs - the desired graphs, depicted in Fig. 9 within a known class of graphs (Laman graphs) that defines all the engineering systems possessing a specific engineering property. In this section it was also shown that the transformation of engineering synthesis problem into a problem in graph theory makes possible use of a known class of graphs that is widely used in a different engineering domain.

In a similar manner, the current section shows that an engineering problem can be transformed not only into a mathematical representation, but also further into another engineering domain. It is first shown (Section 4.3.1) how to transform every linkage in kinematics into a determinate truss in statics thus making it possible to search for the desired topology of the linkage in the domain of statics, as is demonstrated in Section 4.3.2. 

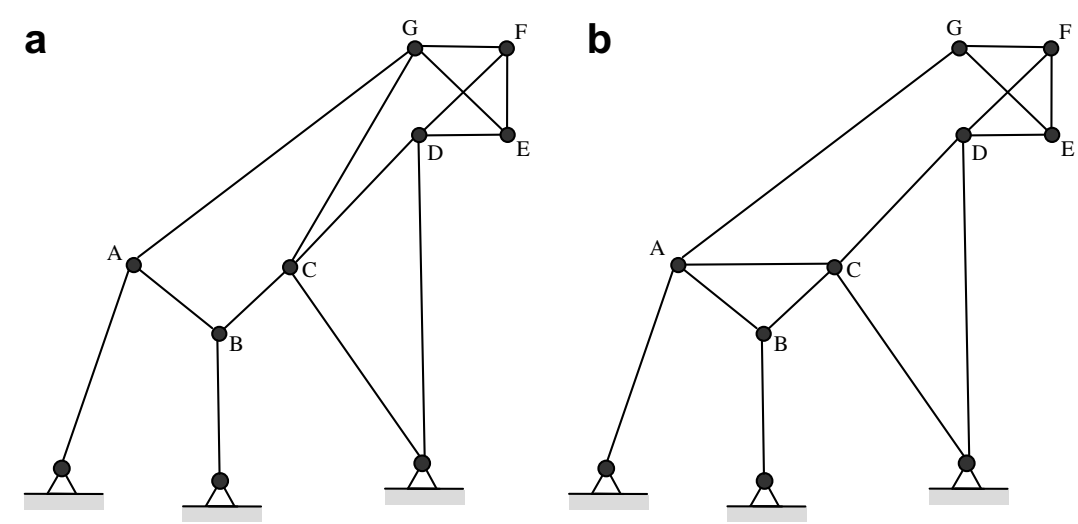

Fig. 10. Example of a framework (a) whose graph topology is Assur and (b) whose graph topology is not Assur.

\subsubsection{Transforming kinematic linkages into static trusses}

The transformation is performed by removing the driving links and replacing them with pinned joints resulting in a determinate truss. For example, the four bar linkage (Fig. 11a) is transformed into a determinate truss by removing the driving link, ' 1 ', and replacing the inner joint A by a fixed support.

\subsubsection{Topology synthesis of linkages with a special locking position property}

The synthesis problem here is to find the topology of linkages that possess special geometries in which the linkages are in locked positions while there exist forces in all the links and the inner joints are mobile. The previous section introduced a methodology for the deterministic transformation between linkages and determinate trusses, which makes it possible to use the knowledge and synthesis methods developed in plane static trusses for synthesis problems in plane kinematic mechanisms.

In this specific synthesis case, based on the results reported in $[22,20]$ the trusses that are Assur graphs, termed Assur trusses, have a special geometry with the self-stress mobility property. Adding a driving link to this specific configuration yields a linkage in which there exist forces in all the links and in which all the joints are infinitesimally mobile, i.e., linkage in the needed locking position.

Fig. 12 depicts the main idea underlying this synthesis case where the linkage (Fig. 12a) is first transformed into a determinate truss (a more compound case than that
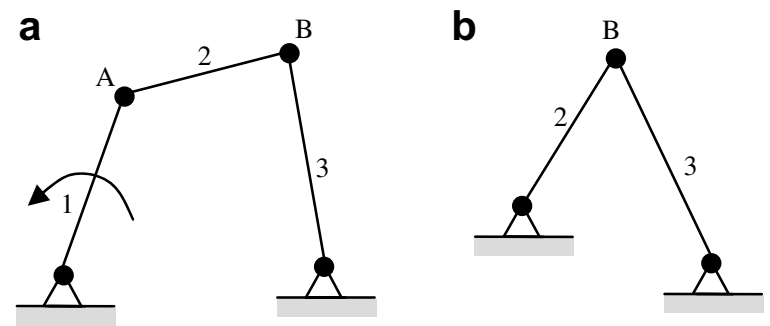

Fig. 11. Example of transforming a linkage (a) into a determinate truss (b). (a) The four bar linkage. (b) The dyad. a

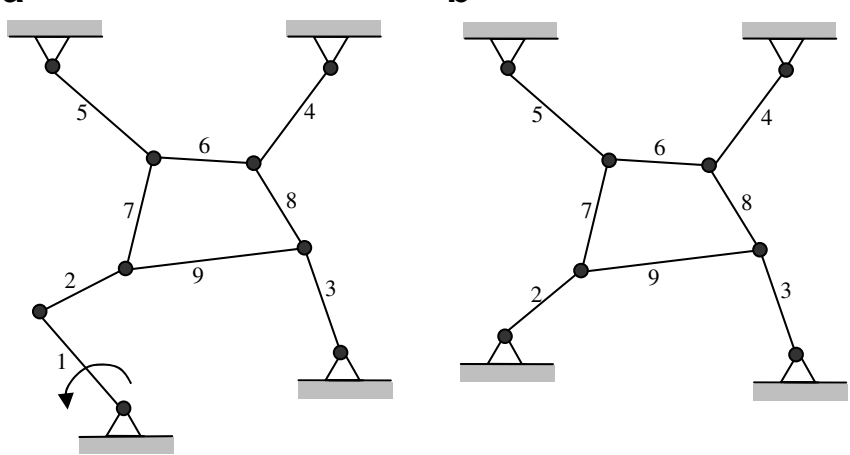

Fig. 12. Example of finding the special locking position in the linkage (a) by finding the self-stress mobility property in the Assur-truss (b).

depicted in Fig. 11), which is then identified as an Assur type determinate truss. It has been mathematically proven that for the latter type of trusses there exists a special configuration which satisfies the desired property - self-stress mobility property.

\section{The topological synthesis process}

In this section the process of deriving the topologies of the systems that possess the desired properties will be described. It is important to notice that although the three synthesis problems are taken from three different engineering domains and are transformed into different graph problems, the methods to produce the graphs are similar.

\subsection{The procedure for obtaining the multi-functional electrical circuits}

The topological procedure is initiated from the minimum self-dual graph consisting of four vertices such that each of its vertices is adjacent to the other three, as shown in Fig. 13a. This graph is called the complete graph with four vertices and is denoted by $\mathrm{K}_{4}$.

The procedure starts by choosing, arbitrarily, one of the vertices to be the ground vertex, (indicated in the 

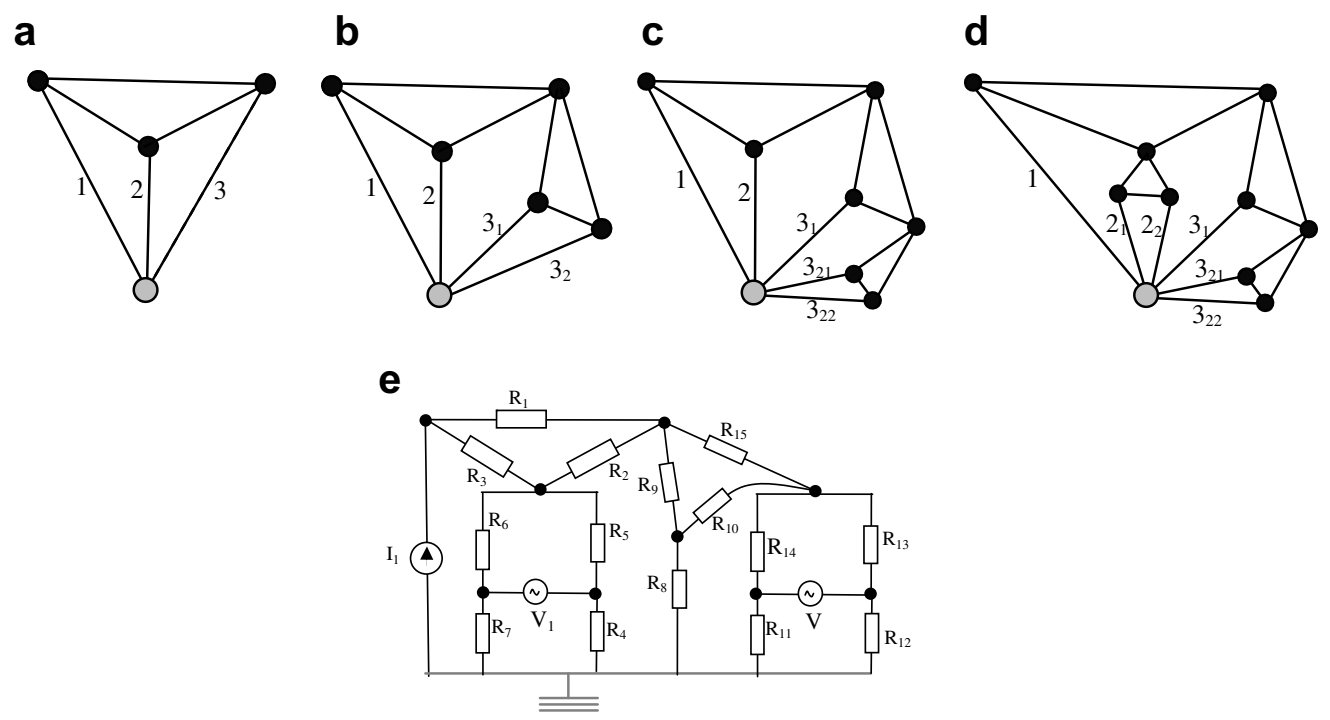

Fig. 13. Example of producing a multi-functional electrical network from the complete graph - K4 (a) The graph $\mathrm{K}_{4}$. (b,c,d) After expanding edges 3 , $3_{1}$, and 2, respectively. (e) The electrical network corresponding to graph (d).

figure as a gray vertex), and all the edges adjacent to this vertex are termed "ground edges". At each step, one of the ground edges is replaced by the complete graph- $\mathrm{K}_{4}$, while the ground edge is deleted, as shown in Fig. 13b. This operation is called expansion [5]. For the sake of clarity, only the grounded edges are marked in Fig. 13 since they are the only edges that can be expanded. It can be proved that any graph obtained through a series of expansions is self-dual, i.e., the graph is isomorphic to its dual graph. The straightforward line of proof of this property is by induction, showing that applying the expansion both on the original and the dual graphs results in identical changes. Since networks, whose corresponding graphs are self-dual, possess the interchangeability property of currents and voltages, in accordance to the result devised in Section 4.1, the procedure of expansion can be employed for constructing multi-functional electrical circuits.

Fig. 13 shows the process of devising the topology of a multi-functional electrical network with 19 elements and 11 junctions.
5.2. Creating the topologies of the trusses with the self-stress mobility property

As was introduced in Section 4.2, only those trusses whose topology is of the Assur graph type possess the selfstress mobility property. Accordingly, the graphs of this type are of value for engineers seeking to design trusses capable of sustaining full self-stresses in all its elements. The current section, therefore, focuses on the process of generating the Assur graphs. The extension process is done by splitting an edge, adding a vertex to the edge, and connecting the new vertex to one of the inner vertices or to the ground. This process can continue till the needed topology is obtained.

Fig. 14 illustrates the process of constructing Assurtrusses from the known triad structure (Fig. 14a).

\subsection{The procedure for creating the linkages with the special locking positions}

Due to the existence of the transformation between plane linkages and trusses, as shown in Section 4.3.1, any
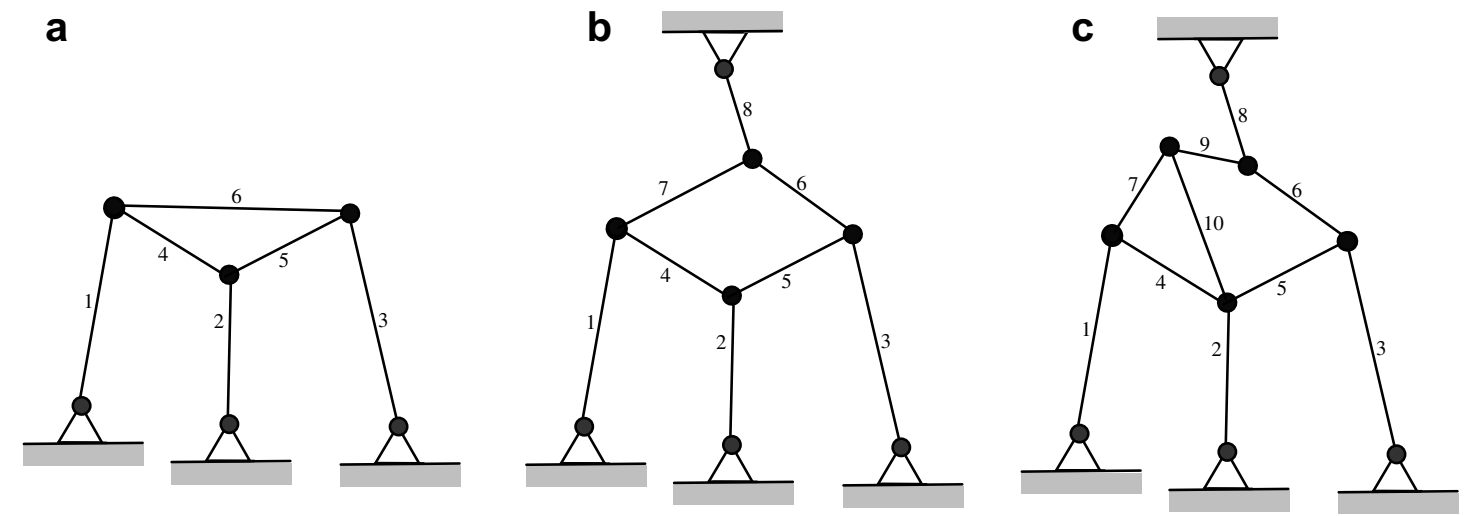

Fig. 14. Example of extending Assur - trusses The trusses in (b) and (c) are obtained after splitting edges 6 and 7 in figures (a) and (b), respectively. 
a

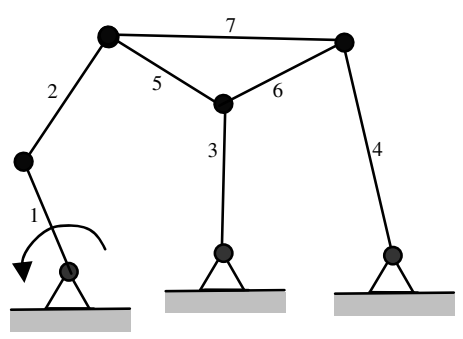

b

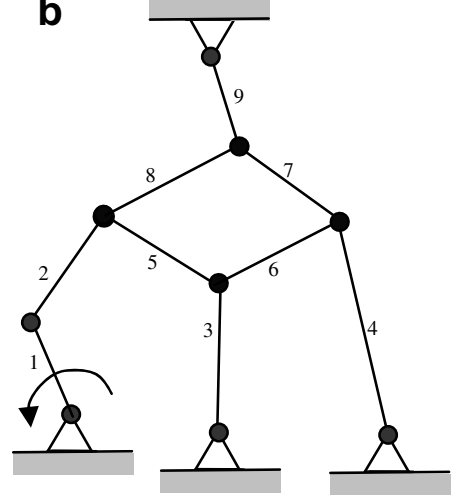

C

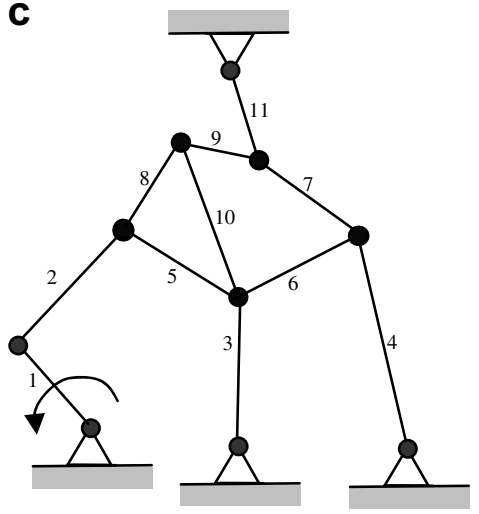

Fig. 15. Examples of linkages which possess the special locking positions derived from the Assur trusses appearing in Fig. 14.

truss with a topology possessing this self-stress and mobility property is transformed (by adding driving links) to a linkage with the special locking positions. Examples of constructing the topology of the linkages by the transformation from statics appear in Fig. 15.

\section{Geometric synthesis step}

In this section, the combinatorial process of finding the configuration for the given linkage topology will be described. Since there is a transformational mapping between linkages and trusses (Section 4.3.1), the example is applied only to a linkage synthesis problem. Electrical networks are one dimensional systems and hence for them this step is not pertinent.

At this stage, the network topology for the linkage has been constructed (Section 5.3). Since it is an Assur graph, it is guaranteed that at least one configuration with the desired property exists. In this section the process of finding such a configuration will be shown.

Due to the duality between trusses and linkages the force vectors in the bars are equal to the dual relative linear velocities in the corresponding links [21]. The proof underlying this relation is the graph theory duality that was employed in Section 4.1.

This duality relation between different graph networks also reveals new concepts and entities. For example, the absolute linear velocity of the joints of the mechanism corresponds to a new variable of force in trusses, termed face force (FF) [21], acting in the corresponding face (a nonbisected contour formed by rods). One of the unique properties of this variable is that the force in the bar is defined by subtraction between its two adjacent face forces as depicted in Fig. 16. This type of variable resembles the known variable in electricity - mesh current which is known to be scalar, while face force is a multidimensional variable. More properties and details on face force can be found in [21,23].

To illustrate and clarify the idea of the duality between trusses and linkages Fig. 17c and d depicts a truss and its

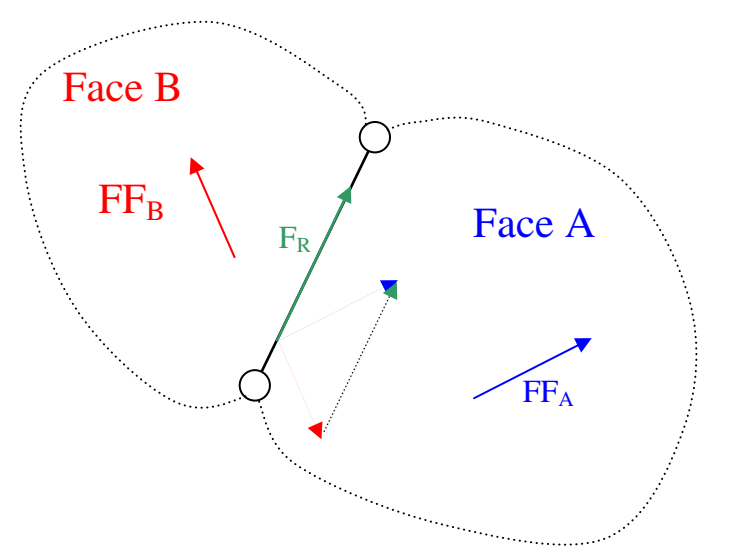

Fig. 16. The force in the rod is defined by its two adjacent face forces.

dual linkage (superimposed) such that the bars and the corresponding links are perpendicular.

The idea underlying the type of a face force variable is illustrated in Fig. 17 by showing the correlation between the voltage of a junction in the original system - the electrical circuit (Fig. 17a) and the mesh current in the dual electrical circuit (Fig. 17b), while Figs. 17c and d highlight the correlation between the linear velocity of a joint and the face force acting in the corresponding face in the dual truss, respectively. This type of physical variable has special properties since it exhibits the qualities of both force and potential (it is the counterpart of the linear velocity). It can have many practical applications, one of which is in the geometric synthesis stage introduced in this paper, as follows:

1. Identify the faces in the linkage.

2. Assign an arbitrary face force vector to each face.

3. Compute the internal link forces from the face force vectors in step 2. For each link the internal force vector is equal to the vector difference between the two face forces associated with the faces separated by this link. Fig. $18 \mathrm{~b}$ provides a vector diagram yielding the internal forces in this manner. In the diagram, the face force vectors found in step 2, are designated by the colored 


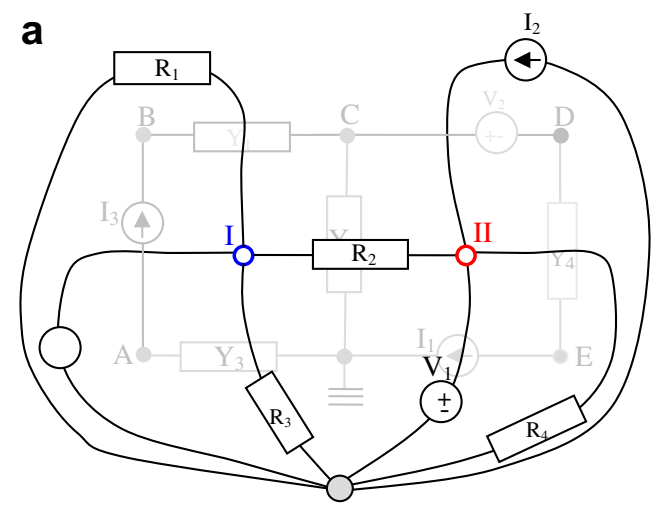

b

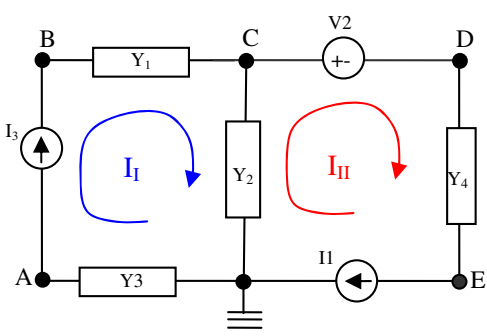

C

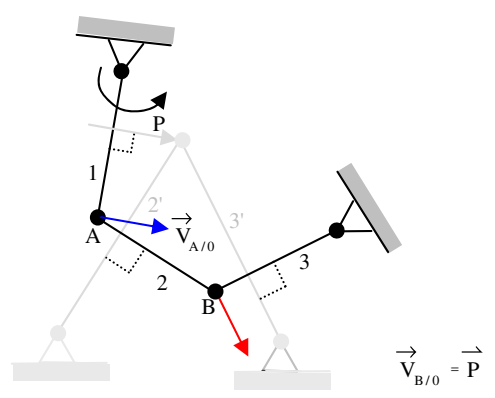

d

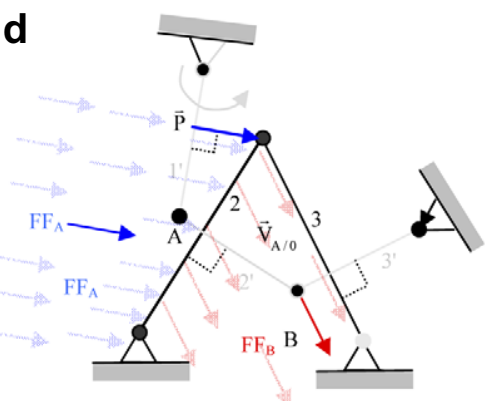

Fig. 17. The duality relation between a potential of a joint and its corresponding variable in the dual system. (a,b) Voltages of electrical circuit junctions and their corresponding mesh currents in the dual electrical circuit. (c,d) The linear velocity of a joint in the linkage and its corresponding face force in the dual determinate truss.
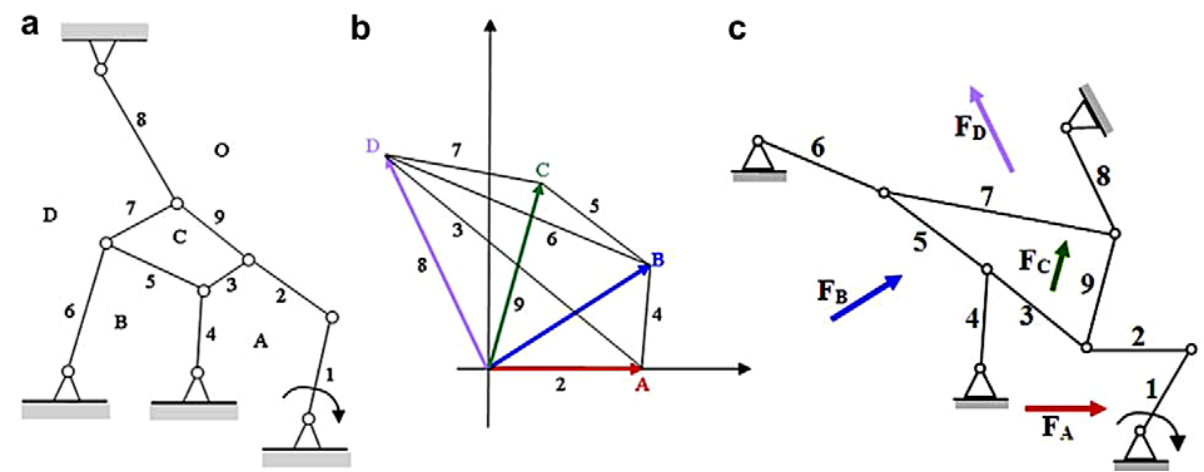

Fig. 18. Example of finding a special configuration using the face-force method. (a) The topology of the linkage. (b) The face-force diagram. (c) The corresponding configuration of the linkage.

arrows starting at the origin, while the internal forces in the links are described by the black lines connecting the two corresponding face forces.

4. In order for the internal link forces obtained in step 3 to correspond to the real physical behavior of the linkage, the angles of the links should be aligned with the angles of their corresponding internal force variables. Thus, redrawing the linkage in accordance with these computed angles yields a geometrical configuration of the linkage for which there exists a feasible set of internal forces. In other words, the linkage configuration obtained through this process is proved to be a linkage in a locked (immobile) position, since it is capable of sustaining the external force applied by the driving links. Fig. 18c shows a link- age whose topology is identical to that of the linkage in Fig. 18a, but the angles of its links are set according to the angles of the vectors obtained from the vector diagram of Fig. 18b. It can be verified through the theorems of machine theory [18] that the linkage configuration of Fig. $18 \mathrm{c}$ is a mechanism in a locked position.

\section{Conclusions}

This paper introduced the potential of employing graph network theory at the initial stage of the synthesis process. The idea was introduced through solving different synthesis problems from different engineering domains: electronics, kinematics and statics. 
The paper highlighted several advantages of using graph networks in synthesis, which are:

Gaining a general perspective on different synthesis problems - the first step in the proposed method (Fig. 1) is to transform the synthesis problem to a problem in terms of graph theory. For example, the problem of synthesis of multi-functional electrical circuits that can switch the behavior between currents and voltages, was transformed into a problem of constructing graphs whose topologies are self-dual. These graphs are well known and are widely used both in theory and practice.

Employing the same graph algorithms for different synthesis problems-the fact that different synthesis problems are transformed into the same graph theory problem opens the possibility of applying the same algorithm for the solution of many analogous problems. Furthermore, it is possible that different synthesis problems are transformed into different graph problems but the same algorithm is used for the latter. This case is reported in the paper where the synthesis of interchangeable electrical circuits was transformed into the synthesis of self-dual graphs (Section 4.1) and the synthesis of trusses with the self-stress mobility property was transformed into Assur graphs (Section 4.2). Although the latter two graph problems are different, the algorithm for their construction is similar, as appears in Section 5.2.

Borrowing knowledge and methods from different engineering disciplines - different graph types correspond to different engineering domains. When there are relations between the corresponding graphs, there is a relation between the engineering domains. For example, due to the relations between the graphs of static systems and those of kinematic systems, a truss possesses the self-stress mobility property if and only if (IFF) the corresponding linkage has a special locking position, as appears in Section 5.3.

The paper also employed a physical variable, referred to as Face Force (Section 6), which, although new to statics, is a multi-dimensional counterpart of the one-dimensional mesh current widely known in electronics.

\section{References}

[1] A. Antonsson, Microsystem design synthesis, in: A. Antonsson, J. Cagan (Eds.), Formal engineering design synthesis, Cambridge University Press, New York, NY, USA, 2001, pp. 126-169.

[2] L.V. Assur, Investigation of planar rod mechanisms from the point of view of their structure and classification, Vol. 20-21, St. Petersburg Polytechnical Institute, Izvestija, 1914.

[3] N. Balabanian, T.A. Bickart, Electrical Network Theory, John Wiley \& Sons, NY, 1969.

[4] M.P. Bendsoe, N. Kikuchi, Generating optimal topologies in structural design using a homogenization method, Computational Methods in Applied Mechanics and Engineering 71 (1988) 197-224.
[5] A.R. Berg, T. Jordan, A proof of Connlelly's conjecture on 3 -connected circuit of the rigidity matroid, Journal of Combinatorial Theory B 88 (1) (2003) 77-97.

[6] M.I. Campbell, J. Cagan, K. Kotovsky, An agent-based approach to conceptual design in a dynamic environment, Research in Engineering Design 11 (1999) 172-192.

[7] M.I. Campbell, J. Cagan, K. Kotovsky, Agent-based synthesis of electromechanical design configurations, AMSE Journal of Mechanical Design 122 (2000) 1-9.

[8] A. Chakrabarti, T.P. Bligh, An approach to functional synthesis of mechanical design concepts: theory, applications, and emerging research issues, Artificial Intelligence in Engineering Design, Analysis, and Manufacturing 10 (1996) 313-331.

[9] M. Chirehdast, H-C. Gea, N. Kikuchi, P.Y. Papalambros, Structural configuration examples of an integrated optimal design process, Journal of Mechanical Design 116 (4) (1994) 997-1004.

[10] N. Deo, Graph Theory with Applications to Engineering and Computer Science, Prentice Hall, 1974.

[11] S.J. Fenves, Structural Analysis by Networks, Matrices and Computers, Journal of the Structural Division, ASCE 92 (1966) 199-221.

[12] C.J. Gantes, Deployable Structures Analysis and Design, Computational Mechanics Inc., 2001

[13] P. Hajela, E. Lee, C.-Y. Lin, Optimal sizing, geometrical and topological design using a genetic algorithm, Structural Optimization 6 (1993) $151-159$.

[14] A. Kaveh, Structural Mechanics: Graph and Matrix Methods, John Wiley \& Sons, 1992.

[15] G. Laman, On graphs and rigidity of plane skeletal structures, Journal of Engineering Mathematics 4 (1970) 331-340.

[16] J. Malmqvist, Computational Synthesis and Simulation of Dynamic Systems, Proceedings of the ASME DTM (1994) 221-230.

[17] J.J. McPhee, On the use of linear graph theory in multibody system dynamics, Nonlinear Dynamics 9 (1996) 73-90.

[18] R.L. Norton, Design of Machinery, McGRAW-HILL, New York, 1992.

[19] P. Papalambros, K. Shea, in: A. Antonsson, J. Cagan (Eds.), Creating Structural Configurations, Cambridge University Press, NY, USA, 2001, pp. 93-125.

[20] B. Servatius, O. Shai, W. Whiteley, Assurance for Assur graphs by rigidity circuits, submitted for the conference book ESI - Program 2006: Rigidity and Flexibility, 2006.

[21] O. Shai, Utilization of the Dualism between Determinate Trusses and Mechanisms, Mechanism and Machine Theory 37 (11) (2002) 13071323.

[22] O. Shai, The Equivalence between Static (rigid) and Kinematic (mobile) Systems through the Graph Theoretic Duality, ESI Program 2006: Rigidity and Flexibility, April 23-May 6, in The International E. Schrödinger Institute for Mathematical Physics, Vienna, Austria, 2006.

[23] O. Shai, G.R. Pennock, A study of the duality between planar kinematics and statics, Journal of Mechanical Design, Spatial Mechanisms and Robot Manipulators, Trans. ASME 128 (3) (2006) $587-598$.

[24] K. Shea, J. Cagan, Innovative dome design: applying geodesic patterns with shape annealing, Artificial Intelligence in Engineering Design, Analysis, and Manufacturing 11 (1997) 379-394

[25] K. Shea, J. Cagan, S.J. Fenves, A shape annealing approach to optimal truss design with dynamic grouping of members, ASME Journal of Mechanical Design 119 (3) (1997) 388-394.

[26] K.T. Ulrich, W.P. Seering, Synthesis of schematic descriptions in mechanical design, Research in Engineering Design 1 (1) (1989) 3-18. 